Flores, P., Ambrósio, J., Claro, J.C.P., Lankarani, H.M., Koshy, C.S., Lubricated revolute joints in rigid multibody systems. Nonlinear Dynamics, Vol. 56(3), 277-295, 2009 (DOI: 10.1007/s11071-008-9399-2)

\title{
Lubricated revolute joints in rigid multibody systems
}

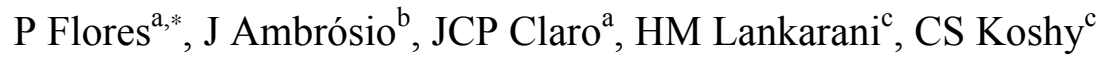 \\ ${ }^{a}$ Department of Mechanical Engineering, University of Minho, Campus de Azurém, 4800-058 \\ Guimarães, Portugal \\ b Institute of Mechanical Engineering, Instituto Superior Técnico, Av. Rovisco Pais 1, 1049-001 Lisboa, \\ Portugal \\ ${ }^{\mathrm{c}}$ Mechanical Engineering Department, Wichita State University, KS 67260-133, USA
}

\begin{abstract}
The main purpose of this work is to present a general methodology for modeling lubricated revolute joints in constrained rigid multibody systems. In the dynamic analysis of journal-bearings, the hydrodynamic forces, which include both squeeze and wedge effects, generated by the lubricant fluid, oppose the journal motion. The hydrodynamic forces are obtained by integrating the pressure distribution evaluated with the aid of Reynolds' equation, written for the dynamic regime. The hydrodynamic forces built up by the lubricant fluid are evaluated from the system state variables and included into the equations of motion of the multibody system. Numerical examples are presented in order to demonstrate the use of the methodologies and procedures described in this work.
\end{abstract}

Keywords: Multibody Dynamics, Lubricated Joints, Hydrodynamic Forces

\footnotetext{
* Corresponding author, Tel: + 351 253510220, Fax: +351 253516007, E-mail: pflores@dem.uminho.pt
} 


\section{Introduction}

In physical joints of mechanical systems clearance, friction and impact are always present, mainly when there is no lubricant fluid. These phenomena can significantly affect the dynamic response of the mechanical systems in so far as the impact causes noise, increases the level of vibrations, reduces the fatigue life of the components and results in loss of precision. When the clearance joints are considered as dry, i.e., without lubricant, contact and friction forces that ultimately cause wear are the main effects present in the physical contact between the surfaces [1-4]. However, in a large number of mechanical systems, the joints are designed to operate with some lubricant fluid, which is an effective way of ensuring better performance of the mechanical systems [5-6].

Journal-bearings are used in many important operating situations, in which the loads vary in both magnitude and direction, often cyclically. Examples include reciprocating machinery such as internal combustion engines, compressors, out of balance rotating machinery such as turbine rotors and other industrial processing machinery. The hydrodynamic fluid film developed in the journal bearings exhibits damping which plays a very important role in the stability of the mechanical elements. In order to study the performance of such journal-bearings, it is necessary to determine the loads and their change in magnitude and direction with time. In dynamically loaded journalbearings the eccentricity and the attitude angle vary through the loading cycle and special care must be taken to ensure that the combination of load and speed rotation does not lead a dangerous small minimum film thickness.

Lubricated joints are designed so that even when the maximum load is applied, the journal and bearing do not come in contact. One of the main reasons for designing journal-bearings in this way is to reduce friction and extend their lifetime.

Consequently, proper modeling of lubricated revolute joints in multibody mechanical 
systems is required to achieve a better understanding of the dynamic performance of the machines. This aspect plays a crucial role owing to the demand for the proper design of the journal-bearings in many industrial applications.

Dynamic analysis of multibody mechanical systems is often conducted under the assumption that bodies are rigid. Material deformation and damping during motion are routinely ignored as well as clearance and lubrication effects [5,7]. Over the last few decades, some authors have been studied planar and spatial mechanical systems with clearance joints [8-10]. Haines [11] derived equations of motion that describe the contributions at an idealized revolute joint with clearance but with no lubrication present. Roger and Andrews [12] developed mathematical models for the journalbearing elements which take into account the effect of clearance, surface compliance and lubrication. However, their lubrication model only accounts for the squeeze-film effect. Later, Liu and Lin [13] extended Roger and Andrews' work to include both squeeze-film and wedge-film actions. Schwab et al. [14], based on the work by Moes et al. [15], applied the impedance method to model lubricated revolute joints in a slidercrank mechanism. Flores et al. [6] proposed a hybrid model for revolute clearance joints in which the dry contact and the pure squeeze-film effects are combined.

To carry out the dynamic analysis of the multibody mechanical systems with lubricated revolute joints, an effective model is developed and presented here. The lubricated revolute joints in mechanical systems deal with force constraints rather than kinematical constraints. In a lubricated revolute joint the journal and bearing act upon each other due to hydrodynamic forces. Therefore, in mechanical systems, a lubricated revolute joint does not involve any kinematic constraint like the ideal joint. Instead, it acts in a similar way to a force element producing time dependent forces. For dynamically loaded journalbearings the classic analysis problem consists of predicting the motion of the journal 
centre under arbitrary and known loading, using, for instance, the mobility method [1618]. Conversely, in the present study the time variable parameters are known from the dynamic analysis and the instantaneous forces on the journal-bearing are calculated. In a simple way, the forces built up by the lubricant fluid are evaluated from the state of variable of the system and included in the equations of motion of the mechanical system. A simple journal-bearing subjected to a constant and unidirectional external load is used as a demonstrative application in order to test different hydrodynamic lubrication models proposed in this work. In addition, results for a planar slider-crank mechanism, in which a lubricated revolute joint in the gudgeon-pin exists, are presented and discussed.

\section{Dynamic characteristics of journal-bearings}

When the journal and bearing have relative angular velocities the amount of eccentricity adjusts itself until the pressure generated in the converging lubricating film balances the external loads. The pressure generated, and, hence, the load capacity of the journalbearing, depends on the journal eccentricity, relative angular velocity, effective viscosity of the fluid lubricant, journal-bearing geometry and clearance. There are two different actions of pressure generation in journal-bearings, namely wedge and squeeze actions, illustrated in Fig. 1. The squeeze action relates the radial journal motion with the generation of load capacity pressure in the lubricant film, whilst the wedge action deals with the relation between relative angular velocity of the journal and bearing ability to produce such pressure. When only the squeeze action of the lubricant is considered, assuming a null or low relative rotational velocity and, hence, absence of relative tangential velocity, the journal load and the fluid reaction force are considered to have the same line of action, which is collinear with the center lines. However, for a more general case, in the presence of high angular velocities, they do not have the same line of action 
because of the wedge effect. When relative angular velocities are large, the simple squeeze approach is not valid and the general Reynolds' equation has to be used $[19,20]$.

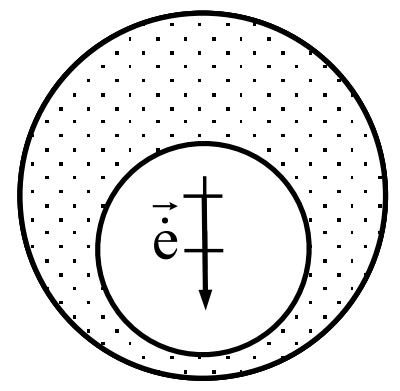

(a)

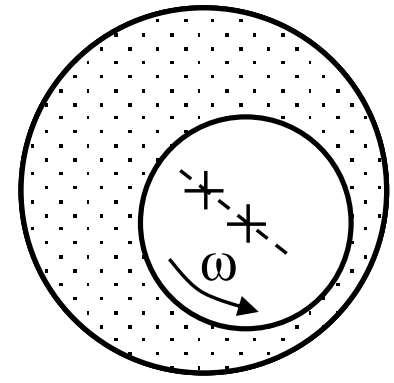

(b)

Fig. 1. (a) Squeeze-film action; (b) Wedge-film action.

In general, mechanical systems demand journal-bearings in which the load varies in both magnitude and direction, which results in dynamically loaded journal-bearings. Figure 2 shows the cross section of a smooth dynamically loaded journal-bearing. When the load acting on the journal-bearing is not constant in direction and/or intensity, the journal center describes an orbit within the bearing boundaries. Typical examples of dynamically loaded journal bearings include the crankshaft bearings in combustion engines and high speed turbines bearings supporting dynamic loads caused by unbalanced rotors.

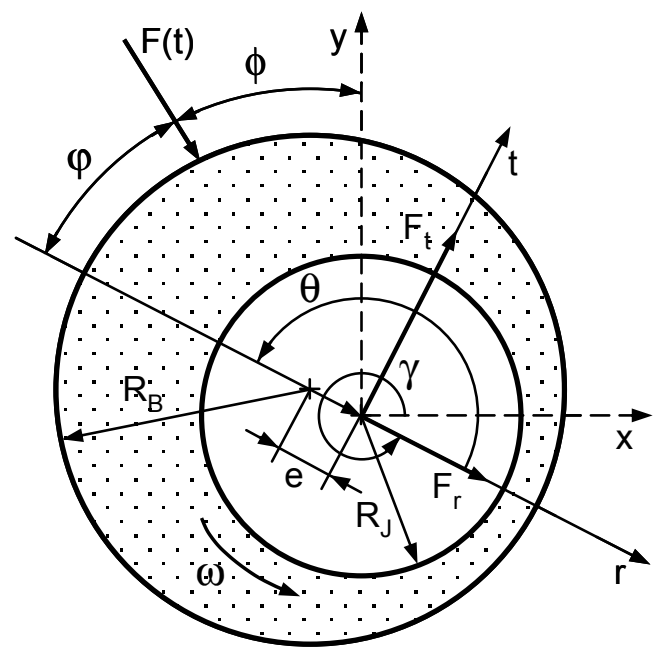

Fig. 2. Cross section of a smooth dynamically loaded journal-bearing. 


\section{Hydrodynamic forces in journal-bearings}

The theory of lubrication for dynamically loaded journal-bearings is mathematically complex and the solution of the governing differential equations is based on many simplifying premises. The main basic principles, terminology and theoretical background are well discussed in the specialized literature, such as Hamrock [19] or Frêne et al. [20]. Pinkus and Sternlicht [21] present a detailed derivation of the Reynolds' equation, in which the forces developed by the fluid film pressure field are evaluated. The Reynolds' equation includes the viscosity, density and film thickness as parameters. The general form of the isothermal Reynolds' equation for a dynamically loaded journal-bearing are written as [21],

$$
\frac{\partial}{\partial X}\left(\frac{h^{3}}{\mu} \frac{\partial p}{\partial X}\right)+\frac{\partial}{\partial Z}\left(\frac{h^{3}}{\mu} \frac{\partial p}{\partial Z}\right)=6 U \frac{\partial h}{\partial X}+12 \frac{d h}{d t}
$$

where $X$ is the radial direction, $Z$ is the axial direction, $\mu$ is the dynamic fluid viscosity, $h$ denotes the film thickness, $p$ is the pressure and $U$ represents the relative tangential velocity between journal and bearing surfaces. The two terms on the right hand side of Eq. (1) represent the two different effects of pressure generation on the lubricant film, i.e., wedge and squeeze actions, respectively. Equation (1) is a non-homogeneous partial differential of the elliptical type. The exact solution of the Reynolds' equation is difficult to obtain and, in general, requires a considerable numerical effort. However, it is possible to solve the equation analytically by assuming null either the first or the second term on the left hand side. These solutions correspond to infinitely-short and infinitely-long journal-bearings, respectively.

Dubois and Ocvirk [22] consider a journal-bearing where the pressure gradient around the circumference is very small when compared with those along the length. This 
assumption is, in general, valid for length-to-diameter $(L / D)$ ratios up to 0.5 . Hence, the Reynolds' equation for an infinitely-short journal-bearing are written as,

$$
\frac{\partial}{\partial Z}\left(\frac{h^{3}}{\mu} \frac{\partial p}{\partial Z}\right)=6 U \frac{\partial h}{\partial X}+12 \frac{d h}{d t}
$$

When the relative pressure is zero at journal-bearing ends the fluid film pressure is [20],

$$
p(\theta, Z)=-\frac{3 \mu}{h^{3}}\left(\frac{L^{2}}{4}-Z^{2}\right)\left((\omega-2 \dot{\gamma}) \frac{\partial h}{\partial \theta}+2 \dot{e} \cos \theta\right)
$$

where $\theta$ is the angular coordinate, $L$ represents the journal-bearing length and $\omega$ is the relative angular velocity between the journal and bearing. The dot in the top on any parameter of Eq. (3) denotes the time derivative of such parameter.

For an infinitely-long journal bearing a constant fluid pressure and negligible leakage in the axial direction are assumed. In many cases it is possible to treat a journal-bearing as infinitely-long and consider only its middle point. This solution was firstly derived by Sommerfeld [23] and is valid for length-to-diameter $(L / D)$ ratios greater than 2 . Thus, the Reynolds' equation for an infinitely-long journal-bearing is,

$$
\frac{\partial}{\partial X}\left(\frac{h^{3}}{\mu} \frac{\partial p}{\partial X}\right)=6 U \frac{\partial h}{\partial X}+12 \frac{d h}{d t}
$$

And the pressure distribution in the fluid is given by [20],

$$
p=6 \mu\left(\frac{R_{J}}{c}\right)^{2}\left\{\frac{(\omega-2 \dot{\gamma})(2+\varepsilon \cos \theta) \varepsilon \sin \theta}{\left(2+\varepsilon^{2}\right)(1+\varepsilon \cos \theta)^{2}}+\frac{\dot{\varepsilon}}{\varepsilon}\left[\frac{1}{(1+\varepsilon \cos \theta)^{2}}-\frac{1}{(1+\varepsilon)^{2}}\right]\right\}
$$

where $R_{J}$ is the journal radius, $c$ is the radial clearance and $\varepsilon$ is the eccentricity ratio.

Equations (3) and (5) enable the calculation of the pressure distributions in hydrodynamic infinitely-short and infinitely-long loaded journal-bearings as functions of the dynamic journal-bearing parameters and geometries. 

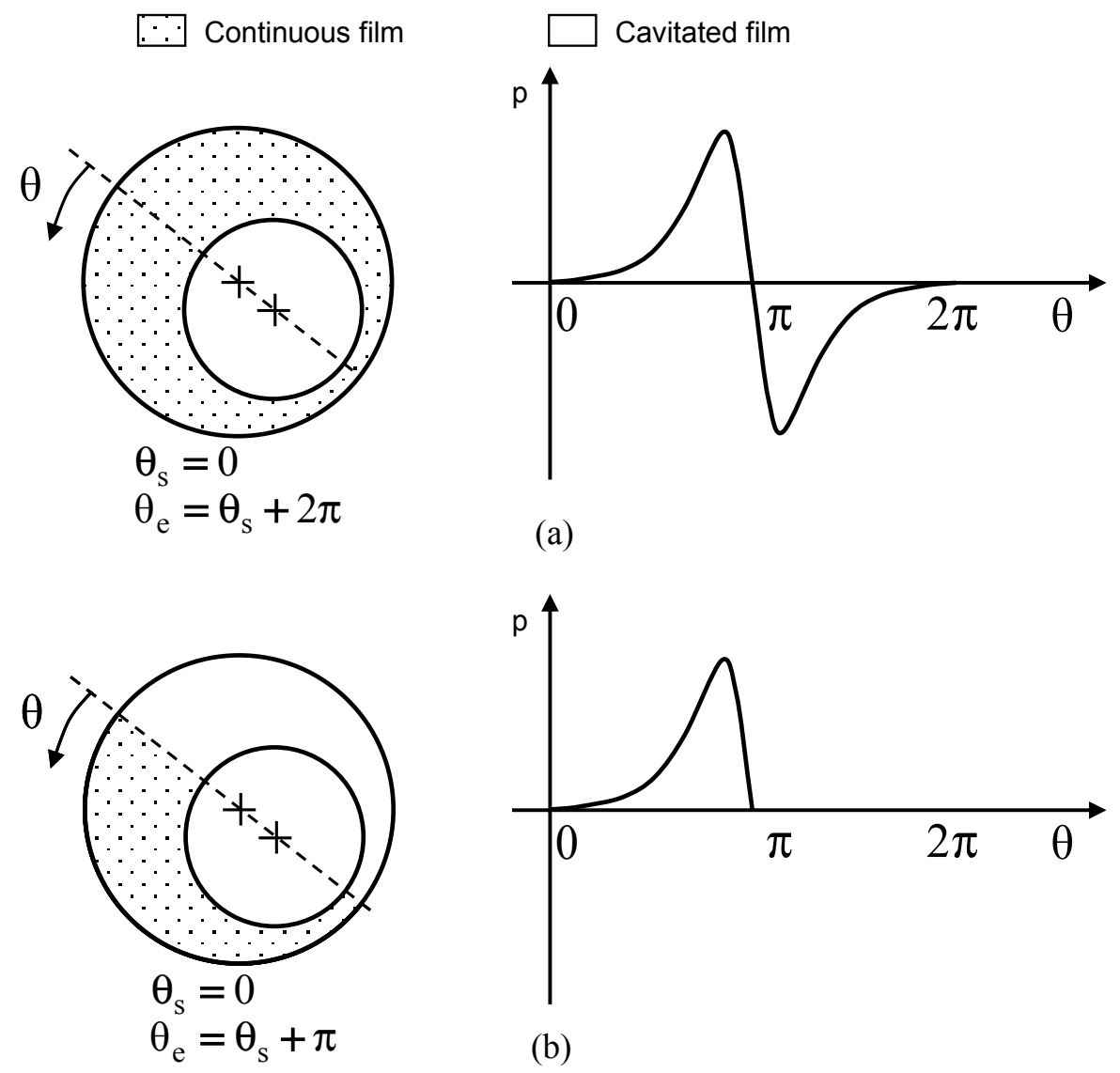

Fig. 3. (a) Sommerfeld's boundary conditions; (b) Gümbel's boundary conditions.

It is convenient to determine the force components of the resultant pressure in directions tangent and perpendicular to the line of centers. These force components can be obtained by integrating the pressure field either in the entire domain $2 \pi$ or half domain $\pi$. In the later case, the pressure field is integrated only over the positive part by setting the pressure in the remaining portion equal to zero. These boundary conditions, associated with the pressure field, correspond to Sommerfeld's and Gümbel's boundary conditions, as illustrated in Fig. 3.

The Sommerfeld's boundary conditions, complete or full film, do not take into account the cavitation phenomenon and, consequently, allow for the existence of negative pressures for the region $\pi<\theta<2 \pi$. This case is not realist in many applications due to the fluid inability to sustain significant sub-ambient pressures. The Gümbel's conditions, 
which account for the film breakdown, take into account the existence of a zero pressure zone for the region between $\pi$ and $2 \pi$. Though the Gümbel's, or half Sommerfeld's solution, results in more realistic predictions of the load capacity, it leads to a violation of the continuity of flow at the outlet end of the pressure curve.

For the Sommerfeld's conditions, i.e., full film, the force components of the fluid film for infinitely-short journal-bearing are written as,

$$
\begin{gathered}
F_{r}=-\frac{\pi \mu L^{3} R_{J}}{c^{2}} \frac{\dot{\varepsilon}\left(1+2 \varepsilon^{2}\right)}{\left(1-\varepsilon^{2}\right)^{5 / 2}} \\
F_{t}=\frac{\pi \mu L^{3} R_{J}}{c^{2}} \frac{\varepsilon(\omega-2 \dot{\gamma})}{2\left(1-\varepsilon^{2}\right)^{3 / 2}}
\end{gathered}
$$

where $F_{r}$ is the radial component of the force while $F_{t}$ is the tangential component, both depicted in Fig. 2.

For the Gümbel's conditions, i.e., film breakdown, the force components of the fluid film for infinitely-short journal-bearing are expressed as,

$$
\begin{gathered}
F_{r}=-\frac{\mu L^{3} R_{J}}{2 c^{2}\left(1-\varepsilon^{2}\right)^{2}}\left(\frac{\pi \dot{\varepsilon}\left(1+2 \varepsilon^{2}\right)}{\left(1-\varepsilon^{2}\right)^{1 / 2}}+2 \varepsilon^{2}(\omega-2 \dot{\gamma})\right) \\
F_{t}=\frac{\mu L^{3} R_{J} \varepsilon}{2 c^{2}\left(1-\varepsilon^{2}\right)^{2}}\left(4 \dot{\varepsilon}+\frac{\pi}{2}(\omega-2 \dot{\gamma}) \sqrt{1-\varepsilon^{2}}\right)
\end{gathered}
$$

As for the case of the infinitely-short journal-bearing, the complete film and the film rupture for the infinitely-long journal-bearing are also distinguished. Thus, for the Sommerfeld's conditions, full film, the force components of the fluid film for infinitelylong journal-bearing are written as,

$$
\begin{gathered}
F_{r}=-\frac{12 \pi \mu L R_{J}^{3} \dot{\varepsilon}}{c^{2}\left(1-\varepsilon^{2}\right)^{3 / 2}} \\
F_{t}=\frac{12 \pi \mu L R_{J}^{3} \varepsilon(\omega-2 \dot{\gamma})}{c^{2}\left(2+\varepsilon^{2}\right)\left(1-\varepsilon^{2}\right)^{1 / 2}}
\end{gathered}
$$


For the Gümbel's conditions the force components of the fluid film for infinitely-long journal-bearing are written as,

$$
\begin{gathered}
F_{r}=-\frac{12 \mu L R_{J}^{3}}{c^{2}}\left(\frac{\varepsilon^{2}(\omega-2 \dot{\gamma})}{\left(2+\varepsilon^{2}\right)\left(1-\varepsilon^{2}\right)}+\frac{\dot{\varepsilon}}{\left(1-\varepsilon^{2}\right)^{3 / 2}}\left(\frac{\pi}{2}-\frac{8}{\pi\left(2+\varepsilon^{2}\right)}\right)\right) \\
F_{t}=\frac{12 \mu L R_{J}^{3}}{c^{2}}\left(\frac{\pi \varepsilon(\omega-2 \dot{\gamma})}{2\left(2+\varepsilon^{2}\right) \sqrt{1-\varepsilon^{2}}}+\frac{2 \varepsilon \dot{\varepsilon}}{\left(2+\varepsilon^{2}\right)\left(1-\varepsilon^{2}\right)}\right)
\end{gathered}
$$

The main difficulty in obtaining satisfactory solutions of journal-bearings dynamics lies not only in solving the differential equations but also in defining adequately the boundary conditions of the Reynolds' equation. In dynamically loaded journal-bearings, the force components, obtained from the integration of the Reynolds' equation only over the positive pressure regions, by assuming null the pressure in the remaining portions, involves finding the zero points, i.e., the angle $\theta_{s}$ for which a position pressure begins and the angle $\theta_{e}$ for which the pressure is null. For the case of a steady-state journal-bearing, these angles are assumed to be equal to 0 and $\pi$, respectively. However, for a dynamically loaded journal-bearing these angles are time dependent and the evaluation of the force components involves a good deal of mathematical manipulation. The details in treatment of these angles are described in the work by Pinkus and Sternlicht [21]. For a positive radial velocity, $\dot{\varepsilon}>0$, the hydrodynamic force components, along the direction of the eccentricity and of its normal, are given by [21],

$$
\begin{gathered}
F_{r}=-\frac{\mu L R_{J}^{3}}{c^{2}} \frac{6 \dot{\varepsilon}}{\left(2+\varepsilon^{2}\right)\left(1-\varepsilon^{2}\right)^{3 / 2}}\left[4 k \varepsilon^{2}+\left(2+\varepsilon^{2}\right) \pi \frac{k+3}{k+3 / 2}\right] \\
F_{t}=\frac{\mu L R_{J}^{3}}{c^{2}} \frac{6 \pi \varepsilon(\omega-2 \dot{\gamma})}{\left(2+\varepsilon^{2}\right)\left(1-\varepsilon^{2}\right)^{1 / 2}} \frac{k+3}{k+3 / 2}
\end{gathered}
$$

For negative radial velocity, $\dot{\varepsilon}<0$, the force components are given by,

$$
F_{r}=-\frac{\mu L R_{J}^{3}}{c^{2}} \frac{6 \dot{\varepsilon}}{\left(2+\varepsilon^{2}\right)\left(1-\varepsilon^{2}\right)^{3 / 2}}\left[4 k \varepsilon^{2}-\left(2+\varepsilon^{2}\right) \pi \frac{k}{k+3 / 2}\right]
$$




$$
F_{t}=\frac{\mu L R_{J}^{3}}{c^{2}} \frac{6 \pi \varepsilon(\omega-2 \dot{\gamma})}{\left(2+\varepsilon^{2}\right)\left(1-\varepsilon^{2}\right)^{1 / 2}} \frac{k}{k+3 / 2}
$$

In Eqs. (14) through (17) the parameter $k$ is defined as,

$$
k^{2}=\left(1-\varepsilon^{2}\right)\left[\left(\frac{\omega-2 \dot{\gamma}}{2 \dot{\varepsilon}}\right)^{2}+\frac{1}{\varepsilon^{2}}\right]
$$

The force components of the resulting pressure distribution for the directions tangent and perpendicular to the line of centers, projected onto the $x$ and $y$ directions, shown in Fig. 2 are given by,

$$
\begin{aligned}
& F_{x}=F_{r} \cos \gamma-F_{t} \sin \gamma \\
& F_{y}=F_{r} \sin \gamma+F_{t} \cos \gamma
\end{aligned}
$$

Equations (6) through (18), for infinitely-short and infinitely-long journal-bearings, present the relation between the journal center motion and the fluid reaction force. The solution of these equations presents no problem since the journal center motion is always known throughout the dynamic analysis of the multibody mechanical system. In traditional tribology analysis of journal-bearings the external forces are known and the motion of the journal center inside the bearing boundaries is evaluated by solving the differential equations for the time dependent variables. However, in the present work instead of the knowledge of the applied load it is the relative journal-bearing motion characteristics that are known and the fluid force is calculated from the pressure distribution in the lubricant. Thus, since all the state of variables of the mechanical system is known from dynamic analysis, the hydrodynamic forces components given by Eqs. (19) are evaluated and included, as external generalized forces, into the system equations of motion of the mechanical system. 


\section{Transition model from hydrodynamic forces to contact forces}

The practical criterion for determining whether or not a journal-bearing is operating satisfactorily is the value of the minimum oil film thickness, which is probably the most important parameter in the performance of the journal-bearings. However, it is not easy to establish a unique value of minimum film thickness that can be assumed to be safe since a great deal depends on the manufacturing process, the alignment of the machine elements associated with the journal-bearings, the general operating conditions, including the environment of the machine, amongst others.

A transition model, which combines the lubricated model and the dry contact model, has been proposed in reference [6] and it overviewed here. Figure 4 shows a partial view of a mechanical system representing a revolute clearance joint with lubrication effect. The parallel spring-damper element represented by a continuous line refers to the solid-to-solid contact between the journal and the bearing wall, whereas, the damper represented by a dashed line is required for the lubricated model.

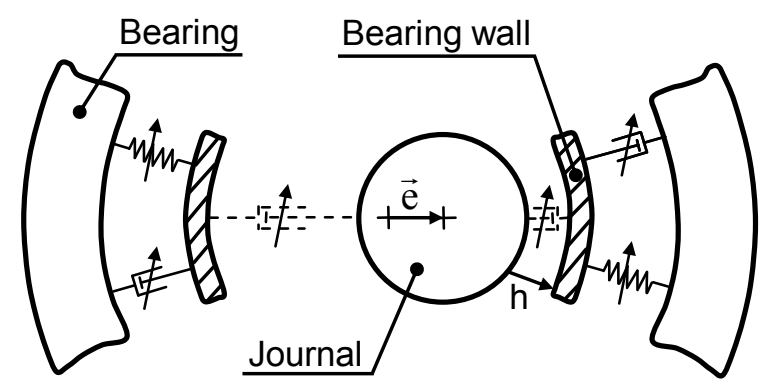

Fig. 4. Mechanical system representing a revolute joint with lubricant effect.

If there is no lubricant between the journal and the bearing, the journal can freely move inside the bearing boundaries. When the gap between the two elements is filled with a fluid lubricant, a viscous resistance force exists and opposes to the journal motion. Since the radial clearance is specified, the journal and bearing can work in two different modes: 
- Mode 1: the journal and the bearing wall are not in contact with each other and they have a relative radial motion. For the journal-bearing model without lubricant, when $e<c$ the journal is in free flight motion and the forces associated to the journal and bearing are null. For lubricated journal-bearing model, the lubricant transmits a force, which must be evaluated from the state variables of the mechanical system, as described before.

- Mode 2: the journal and bearing wall are in contact, thus the contact force between the journal and the bearing is modeled as nonlinear Hertz contact law with a hysteresis damping factor, as described in reference [3].

In short, for a lubricated revolute joint when the film thickness decreases to the thickness of the boundary layer the model switches from mode 1 to mode 2 and the procedure for the dry contact model is used. After the journal changes direction and the bearing wall deformation return to zero, the model switches back to mode 1 .

Since an elasto-hydrodynamic pressure profile is similar to the Hertzian pressure distribution, it is reasonable to change from squeeze-film action, the hydrodynamic lubrication regime, to pure dry contact model. In order to avoid numerical instabilities and to ensure a smooth transition from lubricated model to dry contact model. When the journal reaches the boundary layer, for which the lubricated theory is not valid, the lubricated force model is being substituted by the dry contact force model, as shown in Fig. 5.

This approach ensures continuity in the joint reaction force when the lubricated force model is switched to dry contact force model. The transition force model is,

$$
F=\left\{\begin{array}{ccc}
F_{\text {lubricated }} & \text { if } & e \leq c \\
\frac{\left(c+e_{0}\right)-e}{e_{0}} F_{\text {lubricated }}+\frac{e-c}{e_{0}} F_{d r y} & \text { if } & c \leq e \leq c+e_{0} \\
F_{d r y} & \text { if } & e \geq c+e_{0}
\end{array}\right.
$$


where $e_{0}$ and $e_{1}$ are given tolerances for the eccentricity. The values of these parameters must be chosen carefully, since they strongly depend on the clearance size. It should be noted that the clearance used for the lubricated force model is not $c$ anymore but it is $c+e_{1}$ instead.

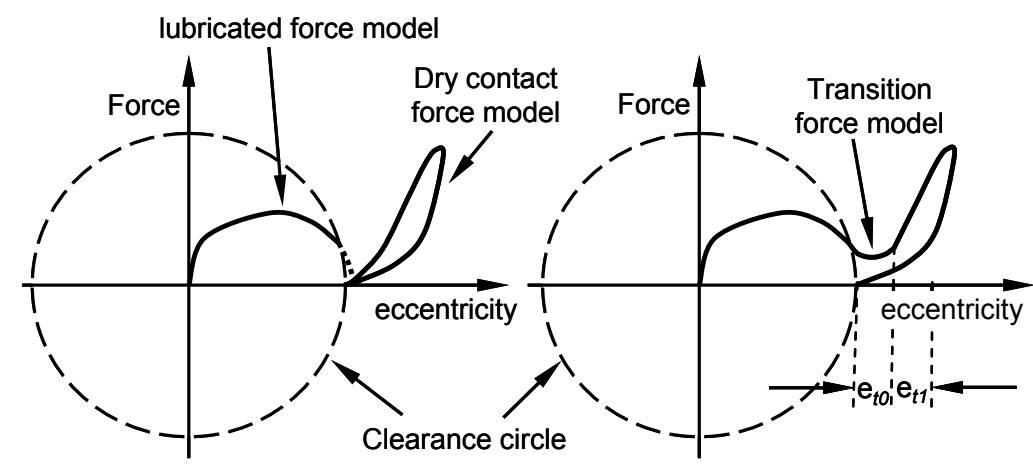

(a)

(b)

Fig. 5. (a) Lubricated and dry contact force models; (b) and transition force model.

\section{Kinematic aspects of journal-bearings interaction}

In order to find the forces produced by the fluid lubricant on the journal-bearings, the different parameters involved in the calculation of these forces need to be evaluated. These hydrodynamic forces are nonlinear functions of the time parameters, $\omega, \varepsilon, \dot{\varepsilon}, \gamma$, and $\dot{\gamma}$, which can be evaluated at any instant of time from the kinematics of the mechanical system.

Figure 6 shows a general configuration of a dynamically loaded journal-bearing in a multibody mechanical system. The two bodies $i$ and $j$ are connected by a lubricated revolute joint, in which the gap between the bearing and the journal is filled with a fluid lubricant. Part of body $i$ is the bearing and part of body $j$ is the journal. The center of mass of body $i$ is $O_{i}$ and the center of mass of body $j$ is denoted by $O_{j}$. Local coordinate systems for bodies $i$ and $j$ are attached to their centers of mass, while a global coordinate 
system is represented by $X Y$. Point $P_{i}$ indicates the center of the bearing and the center of the journal is referred by point $P_{j}$. The coordinate system $X^{\prime} Y^{\prime}$ is parallel to the body fixed coordinate system $(\xi \eta)_{i}$ with its origin in the bearing center.

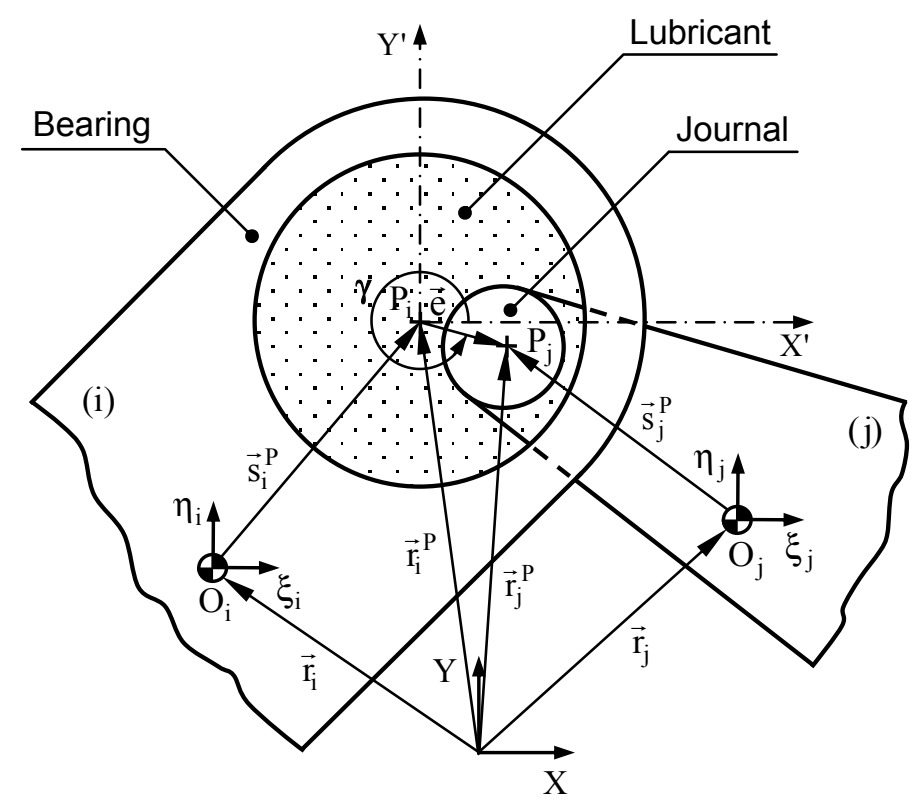

Fig. 6. Generic configuration of a dynamically loaded journal-bearing in a multibody system.

Based on Fig. 6, the eccentricity vector $\mathbf{e}$, which connects the centers of the bearing and journal, is calculated as,

$$
\mathbf{e}=\mathbf{r}_{j}^{P}-\mathbf{r}_{i}^{P}
$$

where both $\mathbf{r}_{j}^{P}$ and $\mathbf{r}_{i}^{P}$ are position vectors written in global coordinates with respect to the inertial reference frame [24],

$$
\mathbf{r}_{k}^{P}=\mathbf{r}_{k}+\mathbf{A}_{k} \mathbf{s}_{k}^{P}, \quad(k=i, j)
$$

The rotational transformation matrix is given by,

$$
\mathbf{A}_{k}=\left[\begin{array}{cc}
\cos \phi_{k} & -\sin \phi_{k} \\
\sin \phi_{k} & \cos \phi_{k}
\end{array}\right]
$$


Variable $\phi_{k}$ represents the angular position of the local coordinate system of body $k$ in the multibody system. Thus, Eq. (21) can be rewritten as,

$$
\mathbf{e}=\mathbf{r}_{j}^{P}+\mathbf{A}_{j} \mathbf{s}_{j}^{\prime P}-\mathbf{r}_{i}^{P}-\mathbf{A}_{i} \mathbf{s}_{i}{ }^{P}
$$

The magnitude of the eccentricity vector is evaluated as,

$$
e=\sqrt{\mathbf{e}^{T} \mathbf{e}}
$$

where $\mathbf{e}^{T}$ is the transpose of vector $\mathbf{e}$.

A unit vector, $\mathbf{r}$, along the eccentricity direction is defined as,

$$
\mathbf{r}=\frac{\mathbf{e}}{e}
$$

The unit radial vector $\mathbf{r}$ is aligned with the line of centers of the journal and bearing.

The tangential direction is defined by rotating vector the radial vector $\mathbf{r}$ by an angle of $90^{\circ}$ in the counter-clockwise direction.

The parameter $\varepsilon$ which defines the eccentricity ratio is the ratio between the distance from the bearing to the journal centers and the radial clearance, that is,

$$
\varepsilon=\frac{e}{c}
$$

The parameter $\dot{\varepsilon}$ is obtained by differentiating Eq. (27) and dividing the result by radial clearance. Thus, differentiating Eq. (27) results in,

$$
\dot{\mathbf{e}}=\dot{\mathbf{r}}_{j}^{P}+\dot{\mathbf{A}}_{j} \mathbf{s}_{j}^{P}-\dot{\mathbf{r}}_{i}^{P}-\dot{\mathbf{A}}_{i} \mathbf{s}_{i}{ }_{i}^{P}
$$

Hence, the time rate of eccentricity ratio is given by,

$$
\dot{\varepsilon}=\frac{\dot{e}}{c}
$$

The line of centers between the bearing and journal makes an angle $\gamma$ with $X^{\prime}$-axis, as shown in Fig. 6. Since the unit radial vector $\mathbf{r}$ has the same direction as the line of centers, the angle $\gamma$ is calculated using the relation, 


$$
\left[\begin{array}{c}
\cos \gamma \\
\sin \gamma
\end{array}\right]=\left[\begin{array}{l}
r_{x} \\
r_{y}
\end{array}\right]
$$

from which,

$$
\gamma=\tan ^{-1} \frac{r_{y}}{r_{x}}
$$

The parameter $\dot{\gamma}$ is obtained by differentiating Eq. (31) with respect to the time, yielding,

$$
\dot{\gamma}=\frac{e_{x} \dot{e}_{y}-\dot{e}_{x} e_{y}}{e^{2}}
$$

The hydrodynamic components of forces of the resulting pressure field projected onto the $\mathrm{X}$ and $\mathrm{Y}$ directions, given by Eq. (19), act on the journal center. Thus, these forces have to be transferred to the centers of mass of the bodies in which bearing and journal are located. Referring to Fig. 7, the hydrodynamic equivalent forces and moments that act on the center of mass of journal body, $O_{j}$, are given by,

$$
\left[\begin{array}{l}
f_{j}^{x} \\
f_{j}^{y} \\
m_{j}
\end{array}\right]=\left[\begin{array}{c}
F_{x} \\
F_{y} \\
-\left(\xi_{j}^{P} \sin \phi_{j}+\eta_{j}^{P} \cos \phi_{j}\right) F_{x}+\left(\xi_{j}^{P} \cos \phi_{j}-\eta_{j}^{P} \sin \phi_{j}\right) F_{y}
\end{array}\right]
$$

The equivalent forces that act on the center of mass of bearing body, at point $O_{i}$ are

$$
\left[\begin{array}{c}
f_{i}^{x} \\
f_{i}^{y} \\
m_{i}
\end{array}\right]=\left[\begin{array}{c}
-F_{x} \\
-F_{y} \\
\left(\xi_{i}^{P} \sin \phi_{i}+\eta_{i}^{P} \cos \phi_{i}+e_{y}\right) F_{x}-\left(\xi_{i}^{P} \cos \phi_{i}-\eta_{i}^{P} \sin \phi_{i}+e_{x}\right) F_{y}
\end{array}\right]
$$

It should be noted that the transport moment produced by transferring the forces from the center of journal to the center of the bearing is given by $m_{T}=e_{y} F_{x}-e_{x} F_{y}$.

\section{Multibody systems' equations of motion}

The position and orientation of a body reference frame is defined, in what follows, by a set of Cartesian coordinates [24]. The position and orientation of rigid body $i$ is defined by, 


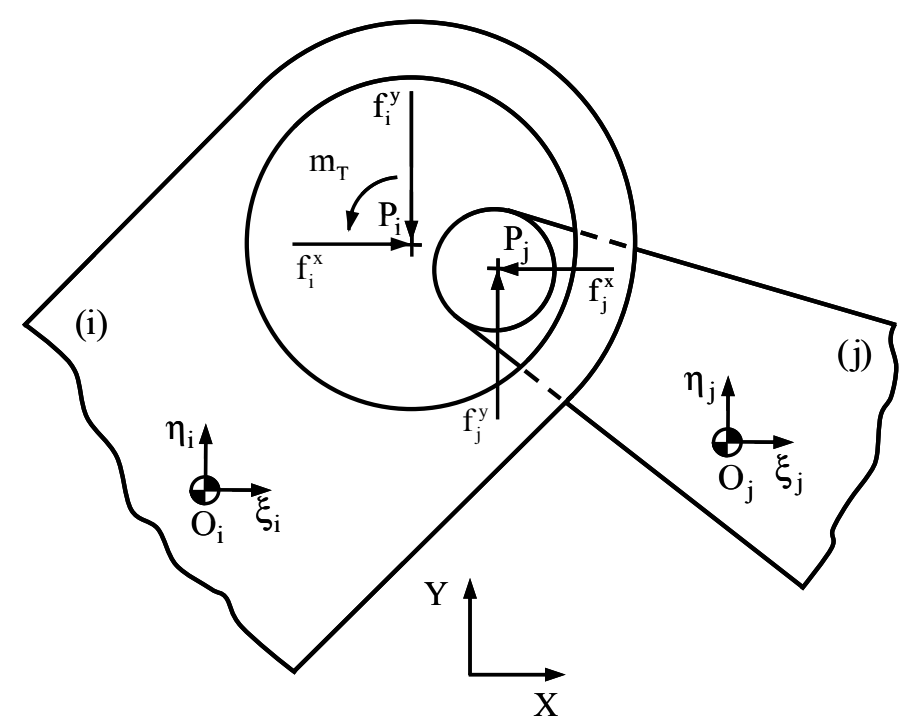

Fig. 7. Hydrodynamic forces acting on the journal and bearing bodies.

$$
\mathbf{q}_{i}^{*}=\left[\begin{array}{ll}
\mathbf{r}_{i}^{T} & \mathbf{p}_{i}^{T}
\end{array}\right]_{i}^{T}
$$

where vector $\mathbf{r}_{i}=\left[\begin{array}{lll}x & y & z\end{array}\right]^{T}$ is the translation of body $i$ and vector $\mathbf{p}_{i}=\left[\begin{array}{llll}e_{0} & e_{1} & e_{2} & e_{3}\end{array}\right]^{T}$ contains the Euler parameters of the body. $i$, which describe its rotation. For computational reasons, it is preferred to use the angular velocities $\boldsymbol{\omega}_{i}^{\prime}$ and angular accelerations $\dot{\boldsymbol{\omega}}_{i}^{\prime}$ of body $i$ instead of the first and second time derivatives of the Euler parameters [24]. The velocities and accelerations of body $i$ are given by vectors,

$$
\begin{aligned}
\dot{\mathbf{q}}_{i} & =\left[\begin{array}{ll}
\dot{\mathbf{r}}_{i}^{T} & \boldsymbol{\omega}_{i}^{\prime T}
\end{array}\right]_{i}^{T} \\
\ddot{\mathbf{q}}_{i} & =\left[\begin{array}{ll}
\ddot{\mathbf{r}}_{i}^{T} & \dot{\boldsymbol{\omega}}_{i}^{\prime T}
\end{array}\right]_{i}^{T}
\end{aligned}
$$

For a constrained multibody system, the kinematical joints are described by a set of holonomic algebraic constraints denoted as,

$$
\boldsymbol{\Phi}(\mathbf{q}, t)=\mathbf{0}
$$

In dynamic analysis of constrained multibody systems, the unique solution is obtained when the acceleration constraint equations are considered simultaneously with the differential equations of motion, for a proper set of initial conditions. Therefore, taking 
the second time derivative of the constraint equations (38) and using the Lagrange multipliers technique, the equations of motion can be written as [24],

$$
\left[\begin{array}{cc}
\mathbf{M} & \boldsymbol{\Phi}_{\mathbf{q}}^{T} \\
\boldsymbol{\Phi}_{\mathbf{q}} & \mathbf{0}
\end{array}\right]\left\{\begin{array}{l}
\ddot{\mathbf{q}} \\
\boldsymbol{\lambda}
\end{array}\right\}=\left\{\begin{array}{l}
\mathbf{g} \\
\boldsymbol{\gamma}
\end{array}\right\}
$$

where $\mathbf{M}$ is the global mass matrix, containing the mass and moment of inertia of all bodies, $\mathbf{g}$ is a force vector that contains the external and Coriolis forces acting on the bodies of the system, $\lambda$ is the vector of Lagrange multipliers and $\gamma$ is the vector that groups all the terms of the acceleration constraint equations that depend on the velocities only, that is,

$$
\boldsymbol{\gamma}=-\left(\boldsymbol{\Phi}_{\mathbf{q}} \dot{\mathbf{q}}\right)_{\mathbf{q}} \dot{\mathbf{q}}-\Phi_{t t}-2 \Phi_{\mathbf{q}} \dot{\mathbf{q}}
$$

The equilibrium equations, represented by Equations (39), do not make an explicitly use of the position and velocity equations associated with the kinematic constraints.

Therefore, due to numerical errors, there is no insurance that the system constraints are fulfilled during the forward dynamic integration of the system velocities and accelerations. To control the constraints violation during numerical integration, the Baumgarte stabilization technique is used, being Equation (39) modified as,

$$
\left[\begin{array}{cc}
\mathbf{M} & \boldsymbol{\Phi}_{\mathbf{q}}^{T} \\
\boldsymbol{\Phi}_{\mathbf{q}} & \mathbf{0}
\end{array}\right]\left\{\begin{array}{l}
\ddot{\mathbf{q}} \\
\lambda
\end{array}\right\}=\left\{\begin{array}{c}
\mathbf{g} \\
\boldsymbol{\gamma}-2 \alpha \dot{\boldsymbol{\Phi}}-\beta^{2} \boldsymbol{\Phi}
\end{array}\right\}
$$

where $\alpha$ and $\beta$ are positive constants that represent the feedback control parameters for the velocity and position constraint violations. The interested reader is referred to Nikravesh [24] for further details on the formulation used and to Baumgarte [25] for details on the stabilization procedures. 


\section{Demonstrative example 1: simple journal-bearing}

A simple journal-bearing subjected to a constant and unidirectional external load, shown in Fig. 8, is used here as a demonstrative example. The journal-bearing under constant unidirectional load contains both the dynamic characteristics within the transient period and the steady hydrodynamic characteristics within the steady-state period that the formulation presented in this work addresses.

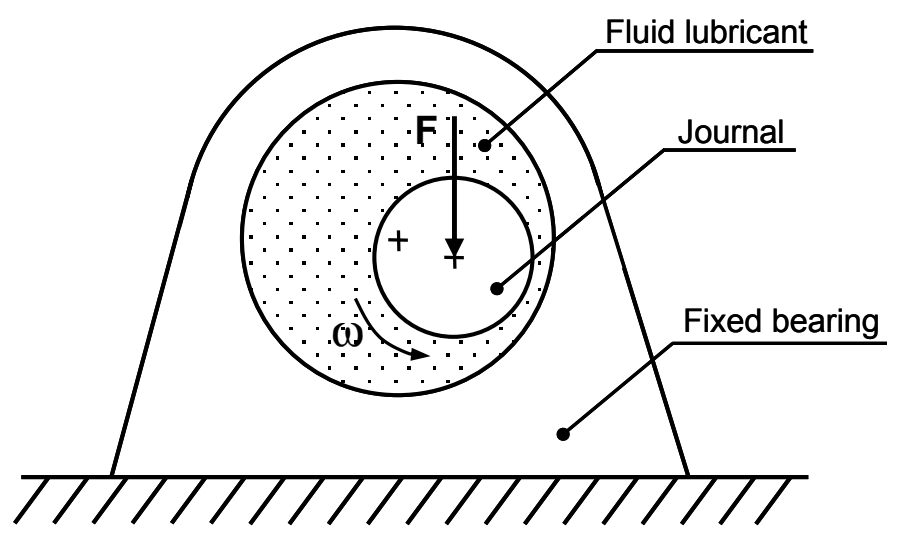

Fig. 8. Simple journal-bearing subjected to a constant external load.

\begin{tabular}{lc} 
Table 1. Dynamic properties of the simple journal-bearing. \\
\cline { 1 - 2 } External load & $30 \mathrm{~N}$ \\
Bearing radius & $10.0 \mathrm{~mm}$ \\
Journal radius & $9.8 \mathrm{~mm}$ \\
Radial clearance & $0.2 \mathrm{~mm}$ \\
Journal-bearing length & $40.0 \mathrm{~mm}$ \\
Journal mass & $0.13 \mathrm{Kg}$ \\
Journal rotational inertia & $2.5 \times 10^{-4} \mathrm{Kgm}^{2}$ \\
Journal angular speed & $500 \mathrm{rpm}$ \\
Oil viscosity at $40^{\circ} \mathrm{C}$ & $400 \mathrm{cP}$ \\
\hline
\end{tabular}

The journal-bearing properties and initial conditions are listed in Table 1. Initially, the journal and bearing centers coincide. The oil fluid used in the present example is a SAE 40 multigrade, which is recommended for small combustion engines and at $40^{\circ} \mathrm{C}$ its viscosity is $400 \mathrm{cP}$. In order to analyze the performance of the models proposed, the 
journal-bearing dynamic response is described by the journal center trajectory inside the bearing boundaries, as represented in Fig. 8, and by the horizontal and vertical components of the fluid force, shown in Figs. 10 and 11, respectively.

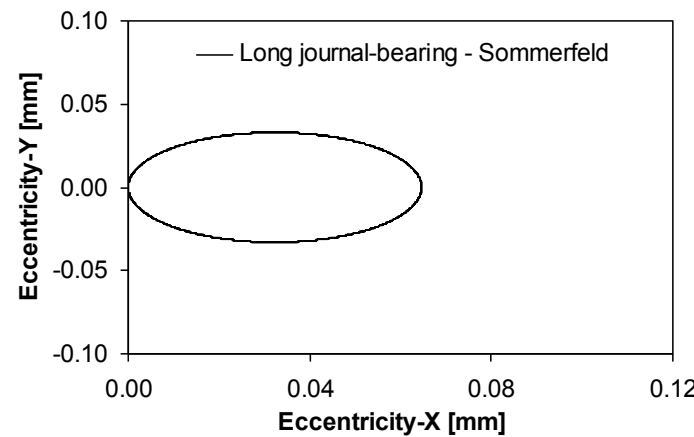

(a)

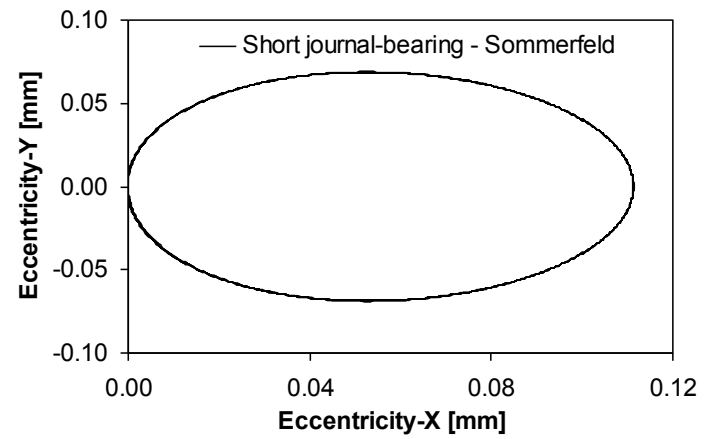

(c)

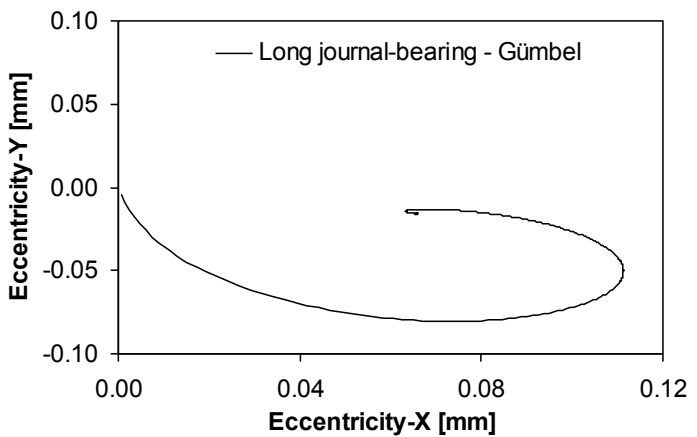

(b)

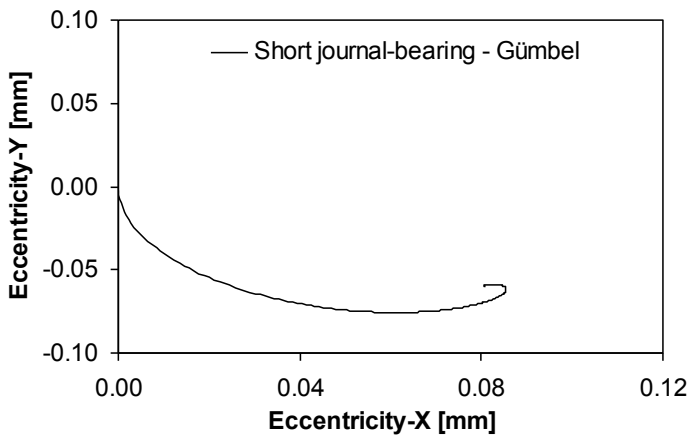

(d)

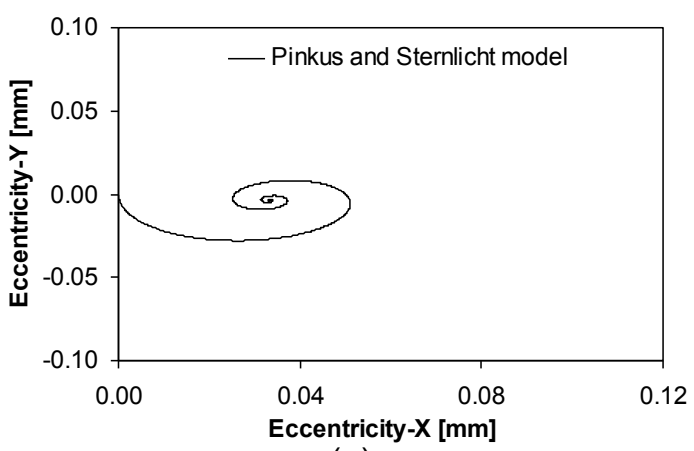

(e)

Fig. 9. Trajectory of the journal center inside the bearing boundaries for the different dynamically loaded journal-bearing models: (a) infinitely-long journal-bearing with Sommerfeld's conditions; (b) infinitely-long journal-bearing with Gümbel's conditions; (c) infinitely-short journal-bearing with Sommerfeld's conditions; (d) infinitely-short journal-bearing with Gümbel's conditions; (e) Pinkus and Sternlicht hydrodynamic model. 


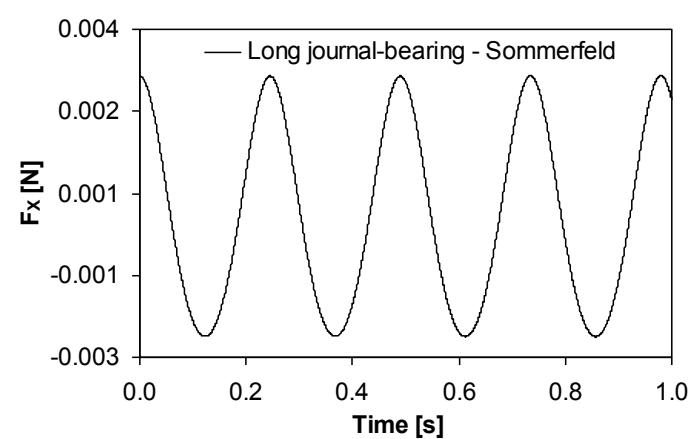

(a)

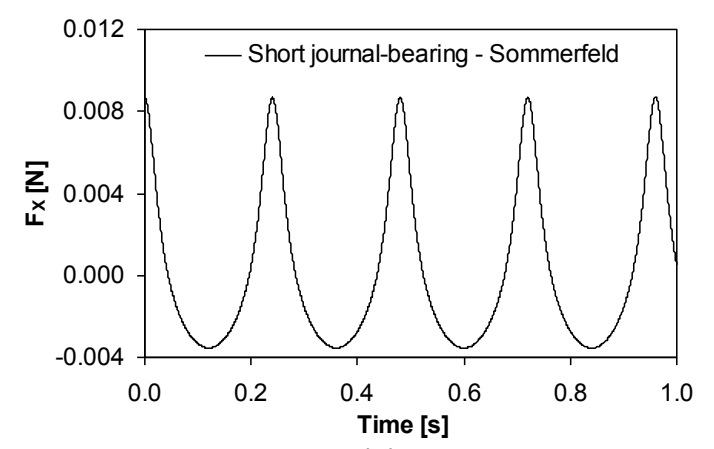

(c)

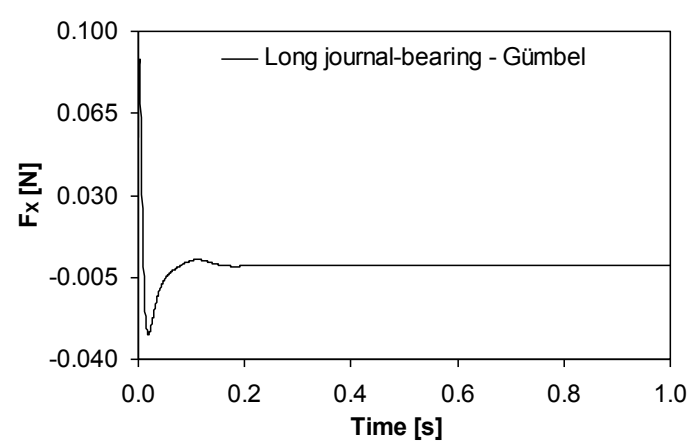

(b)

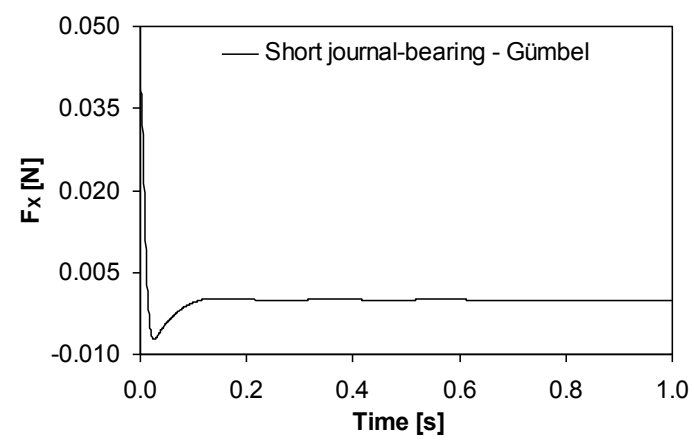

(d)

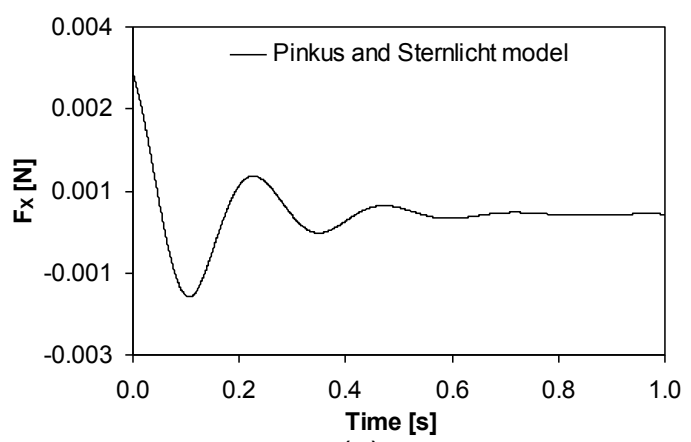

(e)

Fig. 10. Horizontal force on the journal for the different dynamically loaded journalbearing models: (a) infinitely-long journal-bearing with Sommerfeld's conditions; (b) infinitely-long journal-bearing with Gümbel's conditions; (c) infinitely-short journal-

bearing with Sommerfeld's conditions; (d) infinitely-short journal-bearing with Gümbel's conditions; (e) Pinkus and Sternlicht hydrodynamic model.

For the hydrodynamic journal-bearing models that use the Sommerfeld's boundary conditions, the journal center oscillates around its equilibrium position, as observed in Figs. 9a and 9c, whereas for the Gümbel's conditions, after an initial overshoot and transient period, the journal reaches its final equilibrium position, displayed in Figs. 9b and $9 \mathrm{~d}$. In the equilibrium position, the squeeze effect becomes null, that is, $\dot{\varepsilon}=0$. Hence, the forces generated by the wedge action balances the external applied load, which in this 
particular situation is $F_{X}=0$, as observed in Figs. $10 \mathrm{~b}$ and $10 \mathrm{~d}$, and $F_{Y}=F$, as shown in Figs. $11 \mathrm{~b}$ and $11 \mathrm{~d}$. This is expected since the steady-state position is reached and the journal rotates about its whirl. When the Pinkus and Sternlicht hydrodynamic model is used, the journal also reaches the final equilibrium position but with lower damping, as presented in Figs. 9e, 10e and 11e. Indeed, this model predicts lower damping and higher fluctuations in the transient phase which seems to be more realistic since in practical cases the oscillation and instability of the journal-bearing are observed.

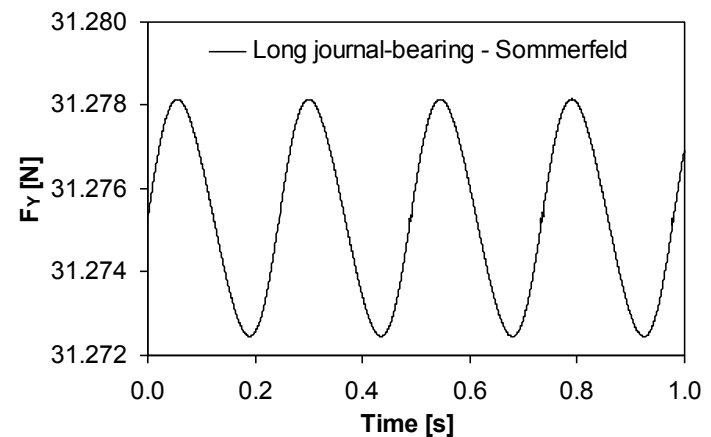

(a)

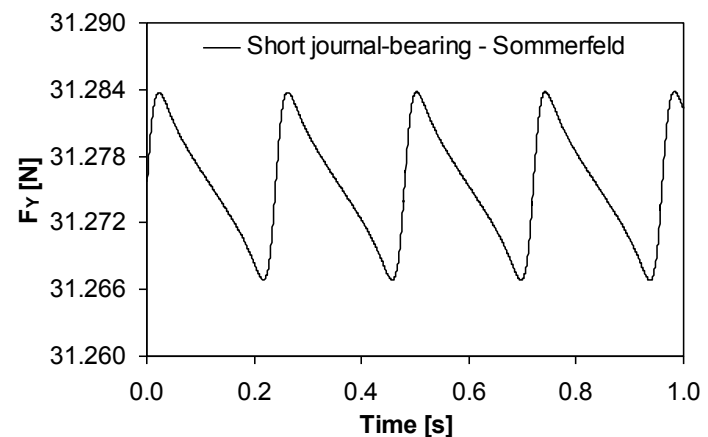

(c)

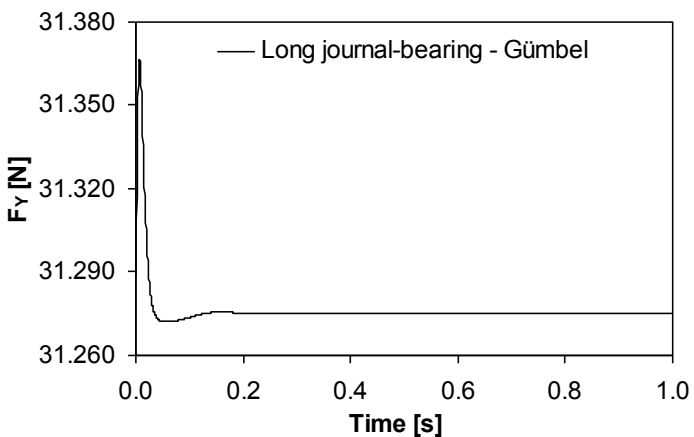

(b)

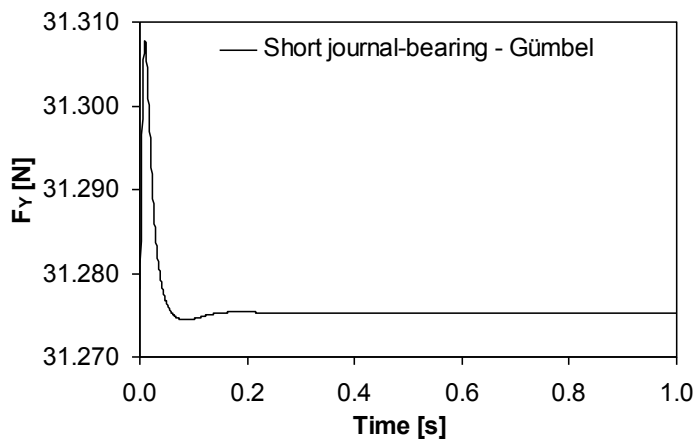

(d)

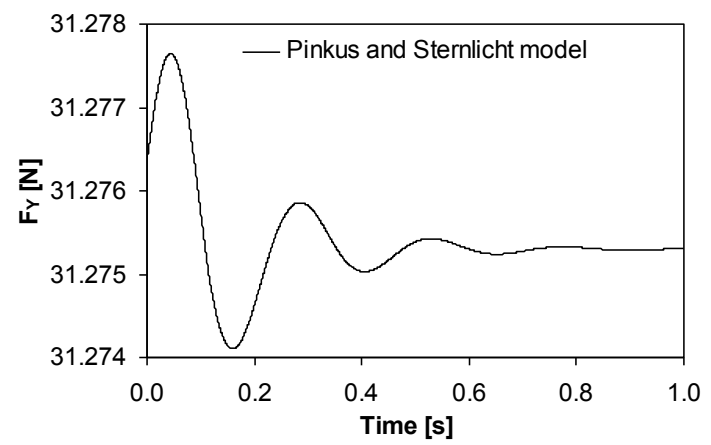

(e)

Fig. 11. Vertical force on the journal for the different dynamically loaded journalbearing models: (a) infinitely-long journal-bearing with Sommerfeld's conditions; (b) infinitely-long journal-bearing with Gümbel's conditions; (c) infinitely-short journalbearing with Sommerfeld's conditions; (d) infinitely-short journal-bearing with Gümbel's conditions; (e) Pinkus and Sternlicht hydrodynamic model. 
From physical point of view a simple journal-bearing subjected to a constant and unidirectional external load corresponds to a free vibrating system. The journal is under the influence of a suddenly applied force, which means that the journal is pulled out from the position of stable equilibrium by a small amount and released. A closer look at the forces displayed in Fig. 10 shows that in all models $F_{X}$ converges or oscillates about $F_{X}=0 N$. By observing Fig. 11 the trend is the convergence or oscillation of $F_{Y}$ about $F_{Y}=31.275 N$.

The journal-bearing performance of the hydrodynamic models with Sommerfeld's boundary conditions corresponds to a free vibration without damping, in which the journal is pulled out of its equilibrium position and then released without initial velocity. The undamped free vibration, being periodic, is represented by a rotating vector, the end of which describes the circle observed in Figs. 9a and 9c.

However, the Gümbel's solutions, shown in Figs. 9b and 9d and the Pinkus and Sternlicht model represent a damped free vibration system. In the later model the end point of the rotating vector describes the logarithmic spiral, displayed in Fig. 9e. The damped cycle path has its pole at the steady-state equilibrium position. In fact, the damped oscillations play an important role in the various forms of hydrodynamic instability and vibration, particularly in lightly loaded journal-bearings. The final equilibrium position clearly depends on the applied load, physical and dynamic properties of the journal-bearing, and on the hydrodynamic model used. The steady-state equilibrium position does not occur in a dynamically loaded journalbearing because the applied load varies both in magnitude and direction. 


\section{Demonstrative example 2: slider-crank mechanism}

In order to illustrate the efficiency and accuracy of the methodology presented in this work, a slider-crank mechanism, with the characteristics described in Table 2 , is used as an application example, as illustrated in Fig. 12. The slider-crank mechanism consists of four rigid bodies, including ground, two ideal revolute joints and one ideal translational joint. A lubricated revolute joint connects the connecting-rod and sliding block. This is an example of a dynamically loaded journal-bearing in so far as the load varies in both direction and magnitude. This revolute joint is modeled with the hydrodynamic model given by Pinkus and Sternlicht [21].

Table 2. Governing properties for the slider-crank mechanism.

\begin{tabular}{cccc}
\hline Body Nr. & Length $[\mathrm{m}]$ & Mass $[\mathrm{Kg}]$ & $\begin{array}{c}\text { Moment of inertia } \\
{\left[\mathrm{Kgm}^{2}\right]}\end{array}$ \\
\hline 2 & 0.05 & 0.30 & $1.0 \times 10^{-4}$ \\
3 & 0.12 & 0.21 & $2.5 \times 10^{-4}$ \\
4 & - & 0.14 & - \\
\hline
\end{tabular}

The performance of the slider-crank mechanism is quantified by plotting the force produced by the fluid on the lubricated joint and the driving crank moment.

Additionally, the journal center orbit inside the bearing limits and the minimum oil film thickness are also plotted. The time interval used corresponds to two complete crank revolutions. The results are compared to those obtained when the system is modeled with all ideal joints.

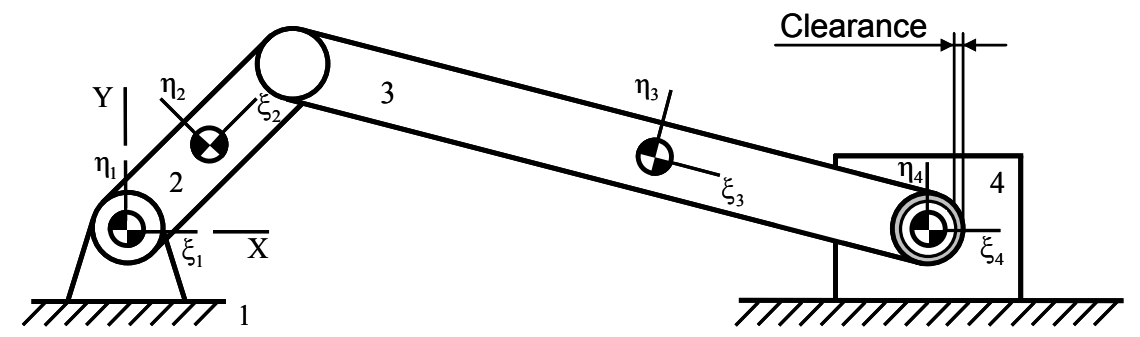

Fig. 12. Slider-crank mechanism with a lubricated revolute joint. 
The crank is driven with a constant angular velocity of $5000 \mathrm{rpm}$. The properties for the lubricated revolute joint are listed in Table 3.

Table 3. Parameters used in the dynamic simulation.

\begin{tabular}{lc}
\hline Bearing radius & $10.0 \mathrm{~mm}$ \\
Journal radius & $9.8 \mathrm{~mm}$ \\
Radial clearance & $0.2 \mathrm{~mm}$ \\
Journal-bearing length & $40.0 \mathrm{~mm}$ \\
Dynamic fluid viscosity & $400 \mathrm{cP}$ \\
\hline
\end{tabular}

Figure 13a shows the force developed in the lubricated joint, that is, the resultant force due to the generation of the pressure field in the journal-bearing, while Fig. 13b depicts the crank reaction moment.

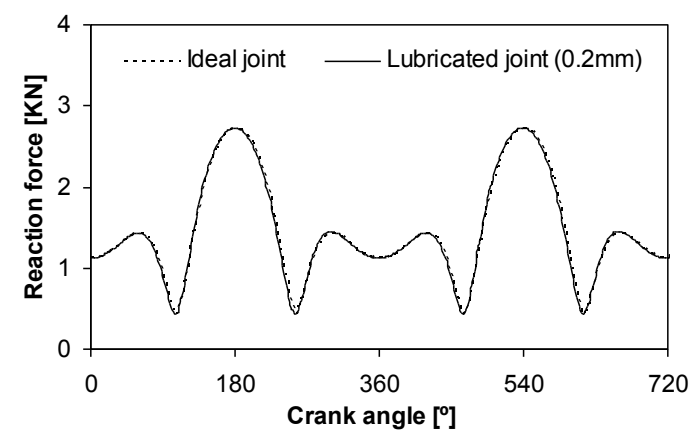

(a)

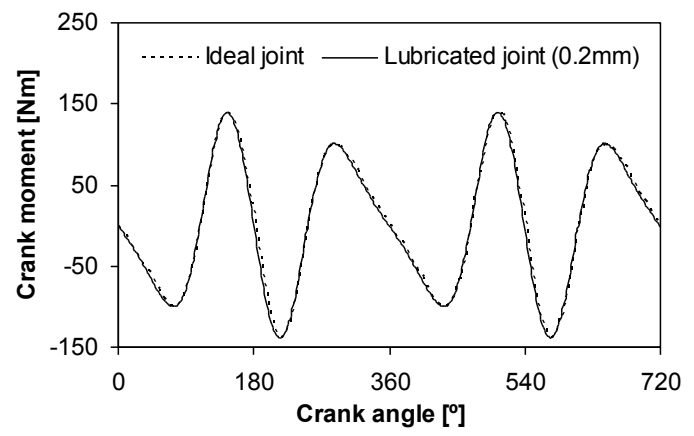

(b)

Fig. 13. (a) Reaction force developed in the lubricated revolute joint; $c=0.2 \mathrm{~mm}$, $\mu=400 \mathrm{cP}$; (b) Driving crank moment; $\mathrm{c}=0.2 \mathrm{~mm}, \mu=400 \mathrm{cP}$.

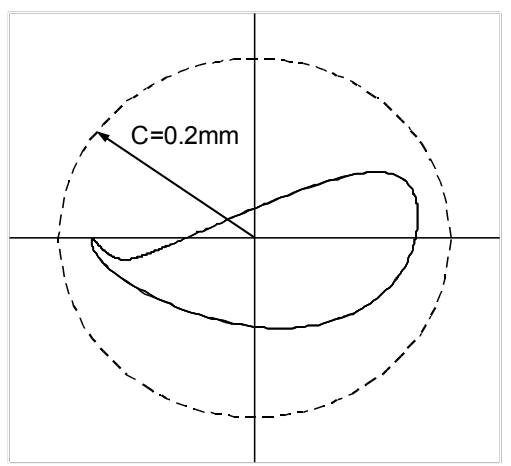

Fig. 14. Journal center orbit inside the bearing; $c=0.2 \mathrm{~mm}, \mu=400 \mathrm{cP}$. 
The observation of Figs. 13a and $13 \mathrm{~b}$ show the global results obtained for the lubricated joint model are of the same order as those obtained with ideal joint. The smooth curve obtained for reaction force is propagated throughout the mechanical system until the crank moment. The first and the second crank rotation show the same results which indicate that the system has reached a steady operation state. This is confirmed by the orbit of the journal center relative to the bearing center, in which the journal moves far away from the bearing wall, meaning that there is always a minimum film lubricant in between the two bodies, as observed in Figs. 14 and 15. Since the load on the journal-bearing under consideration is not constant in direction and magnitude, the journal center describes a trajectory within the bearing boundaries, displayed in Fig. 14. This means that the steady-state equilibrium is not reached, which results in a time dependent loci of the journal center inside the bearing.

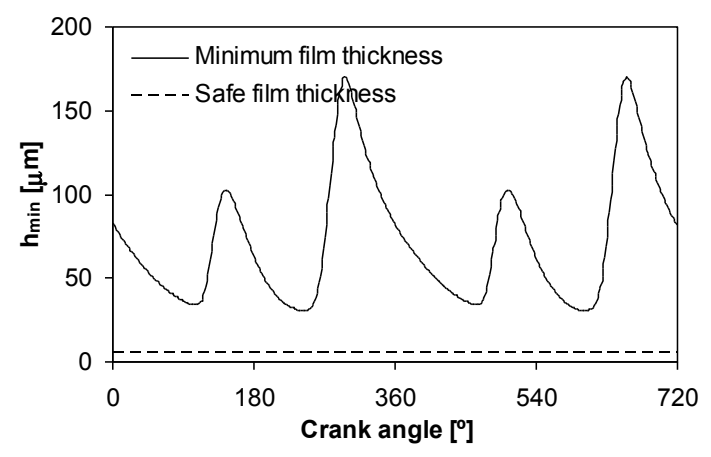

Fig. 15. Minimum and safe oil film thickness; $c=0.2 \mathrm{~mm}, \mu=400 \mathrm{cP}$.

The practical criterion for determining whether or not a journal-bearing is operating satisfactorily is the value of the minimum film thickness. The minimum film thickness for an aligned journal-bearing is given by,

$$
h_{\text {min }}=c(1-\varepsilon)
$$

where $\varepsilon$ is the eccentricity ratio and $c$ is the radial clearance. For safe journal-bearings performance, a minimum film thickness is required. The safe allowable film thickness 
depends on the surface finish of the journal. Hamrock [19] suggests that the safe film thickness should be greater then $2.5 \mu \mathrm{m}$. In practical engineering design it is recommended that the safe film thickness should be at least $0.00015 \mathrm{~mm} / \mathrm{mm}$ of bearing diameter [26]. Thus, for the journal-bearing considered here, the safe film thickness that ensures good operating conditions is of order of $3 \mu \mathrm{m}$ and it is represented in Fig. 15. A similar value for the minimum film thickness is obtained using the ESDU 84031 Tribology series design criterion [27].

From Eqs. (14) through (18) it is clear that the parameters that influence the journalbearing performance are the oil viscosity $\mu$, radial clearance $c$, bearing length $L$, journal radius $R_{J}$ the dynamic journal-bearing parameters $\omega, \varepsilon, \dot{\varepsilon}, \gamma$, and $\dot{\gamma}$. Since the dynamic parameters of the journal-bearing depend directly on the system configuration, the radial clearance size and the oil viscosity are the only possible variables. Several simulations of the slider-crank mechanism for different values of clearance size and oil viscosity are performed to understand the effect of these parameters on its dynamic response. The behavior of the mechanism is quantified by measuring the values of joint reaction force, driving crank moment and journal center trajectory. Values for the radial clearance size of 0.5 and $0.1 \mathrm{~mm}$ and for the oil viscosity of 400 and $40 \mathrm{cP}$ are used in several simulations.

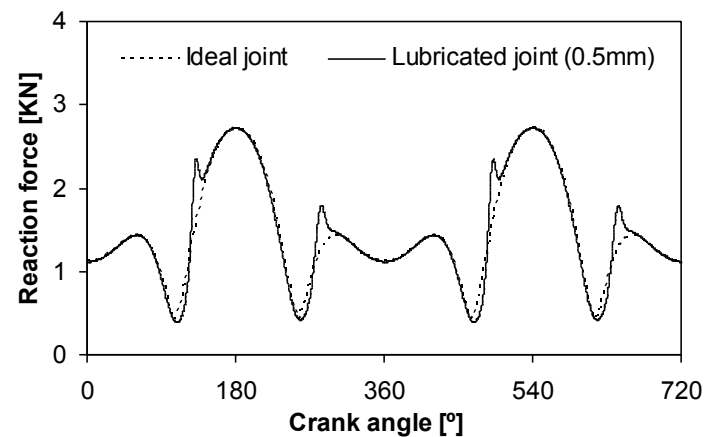

(a)

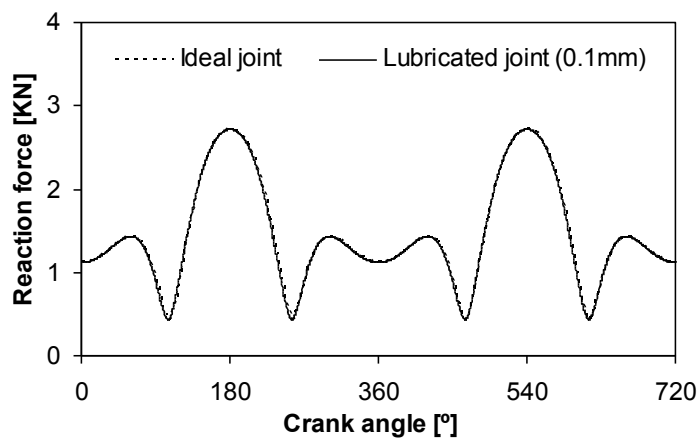

(b)

Fig. 16. Reaction force in the lubricated revolute joint: (a) $c=0.5 \mathrm{~mm}, \mu=400 \mathrm{cP}$; (b) $\mathrm{c}=0.1 \mathrm{~mm}, \mu=400 \mathrm{cP}$. 


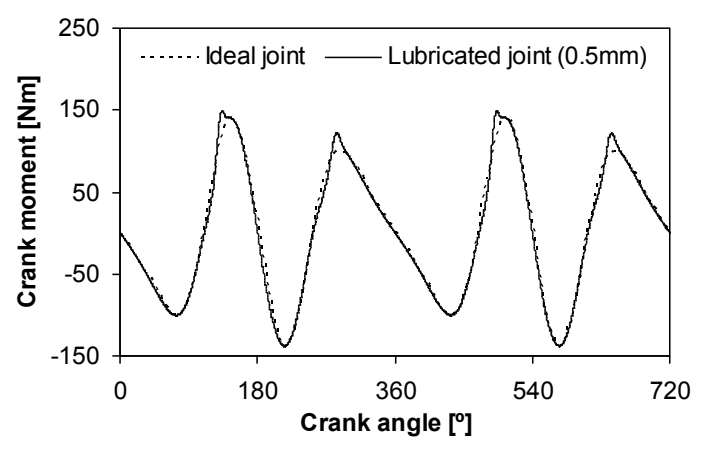

(a)

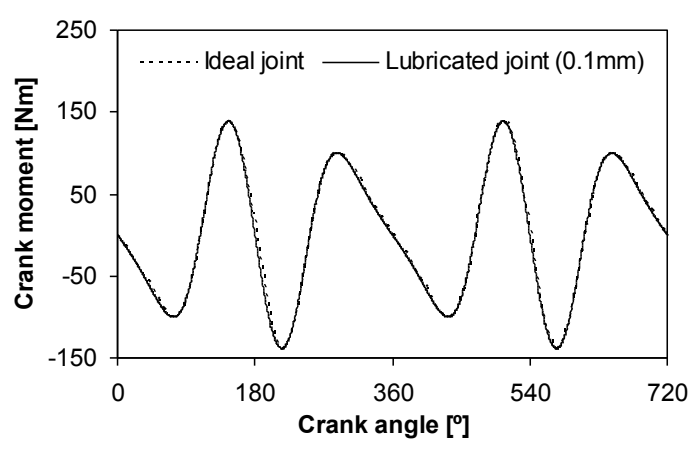

(b)

Fig. 17. Driving crank moment: (a) $c=0.5 \mathrm{~mm}, \mu=400 \mathrm{cP}$; (b) $\mathrm{c}=0.1 \mathrm{~mm}, \mu=400 \mathrm{cP}$.

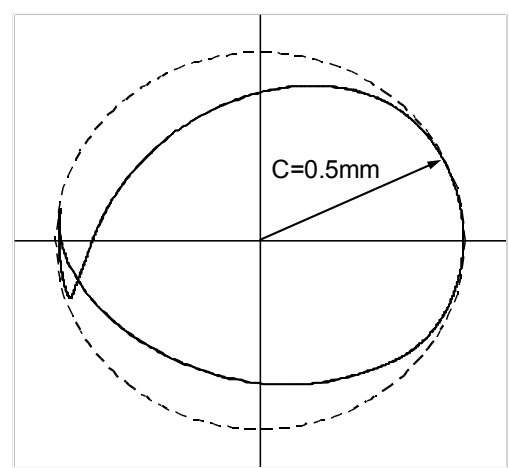

(a)

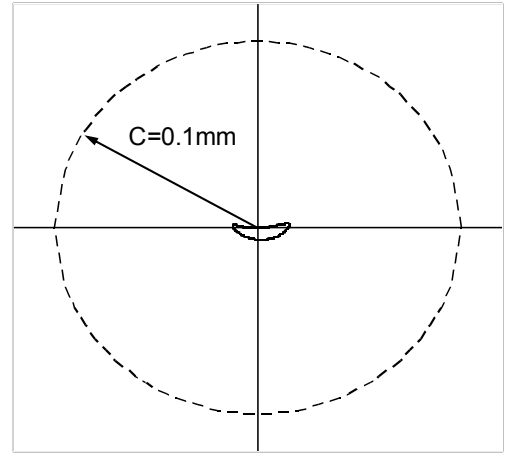

(b)

Fig. 18. Journal center trajectory inside the bearing: (a) $\mathrm{c}=0.5 \mathrm{~mm}, \mu=400 \mathrm{cP}$; (b) $\mathrm{c}=0.1 \mathrm{~mm}, \mu=400 \mathrm{cP}$.

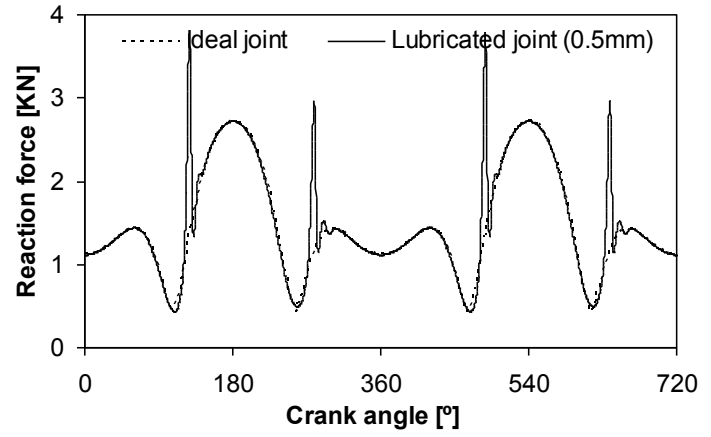

(a)

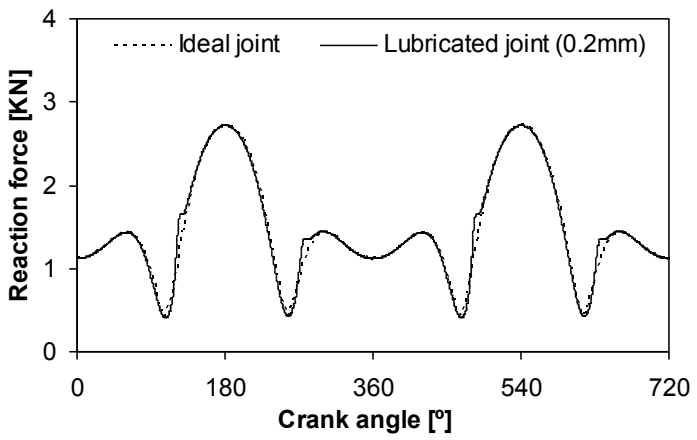

(b)

Fig. 19. Reaction force developed in the lubricated revolute joint: (a) $\mathrm{c}=0.5 \mathrm{~mm}$, $\mu=40 \mathrm{cP}$; (b) $\mathrm{c}=0.2 \mathrm{~mm}, \mu=40 \mathrm{cP}$. 


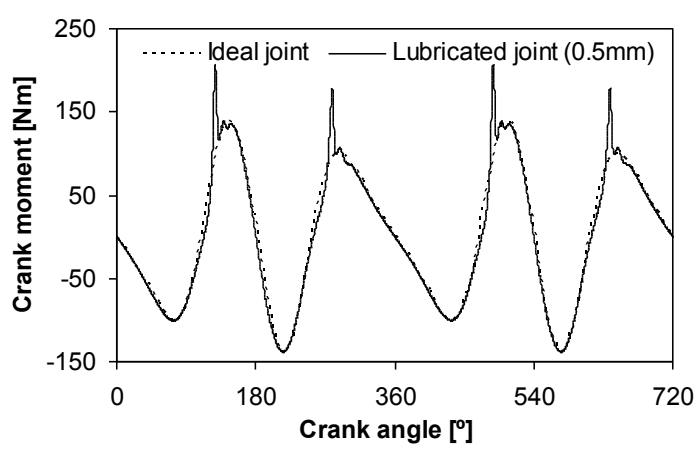

(a)

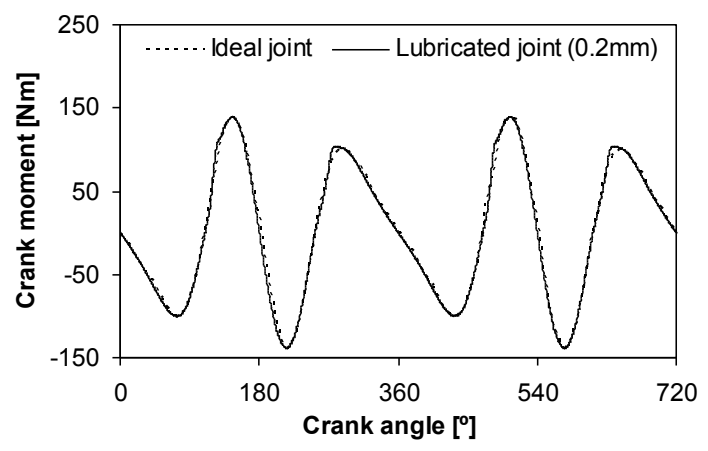

(b)

Fig. 20. Driving crank moment: (a) $c=0.5 \mathrm{~mm}, \mu=40 \mathrm{cP}$; (b) $c=0.2 \mathrm{~mm}, \mu=40 \mathrm{cP}$.

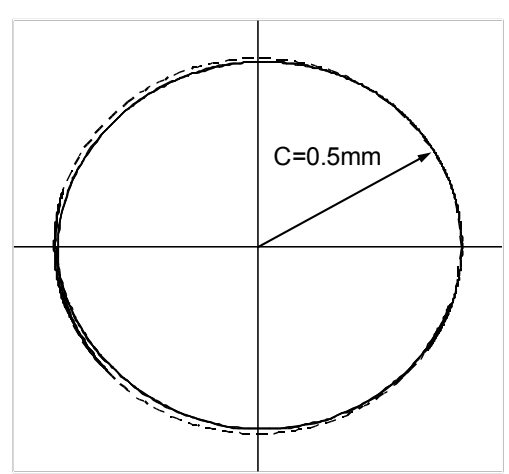

(a)

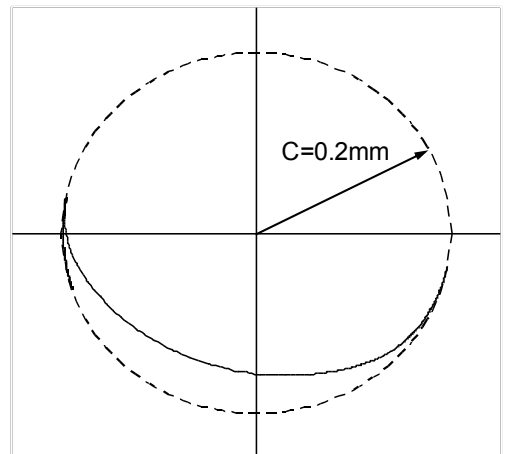

(b)

Fig. 21. Journal center trajectory inside the bearing: (a) $c=0.5 \mathrm{~mm}, \mu=40 \mathrm{cP}$; (b) $\mathrm{c}=0.2 \mathrm{~mm}, \mu=40 \mathrm{cP}$.

There are some important differences between the results obtained for different cases, namely in what concerns the radial clearance size and oil viscosity influence on the joint reaction force and crank moment, such differences are graphically displayed in Figs. 16 through 21. The journal-bearing clearance is an important factor for the satisfactory operation of the journal-bearings. Small values of clearance can give rise to high journal-bearing temperatures while large values of clearance can mean excessive lubricant flow rates. Furthermore, the results clearly show the sensitivity of the system response with different values of viscosity. As expected, with low viscosity the journal and the bearing walls are closer than what is observed for high viscosity, which suggests 
the possibility of metal-to-metal contact, especially visible in Figs. 19 through 21.

Moreover, there are some numerical instabilities associated with the lubricated model, namely when the oil viscosity is low, the clearance is too large or for a combination of these two factors. These numerical difficulties are well represented by some peaks in the joint reaction force and crank moment diagrams, because the journal and bearing walls are very close to each other. When the two elements are very close, the hydrodynamic lubrication theory is no longer valid and the elastohydrodynamic lubrication theory must be taken into account [28,29].

\section{Summary and concluding remarks}

A general methodology for modeling and evaluating the forces produced by a dynamic journal-bearing in multibody systems has been presented and demonstrated in this work. A simple journal-bearing subjected to a constant and unidirectional external load was used as a simple example to test the different hydrodynamic lubrication models. In addition, results for a planar slider-crank mechanism, in which a lubricated revolute joint in the gudgeon-pin exists, were presented and discussed.

In an application for slider-crank mechanism, the reaction moment necessary to drive the crank with a constant angular velocity is of the same order as the case of an ideal joint, meaning that the use lubricant at the machine joints is an effective way of ensuring better performance. The crank moment required to maintain constant the crank angular velocity is a smooth function of time, meaning that the global motion of the slider-crank mechanism with a lubricated joint is periodic or regular. The lubricant acts like a nonlinear spring-damper in so far as lubricated journal-bearing absorbs some of the energy produced by the slider when it accelerates or decelerates, which results in lower reaction moments when compared to ideal joints. Indeed, the lubricant introduces 
effective stiffness and damping to the slider-crank mechanism. A hydrodynamic fluid film journal bearing exhibits a damping effect that plays a very important role in the stability of this kind of journal-bearing.

The hydrodynamic model for lubricated revolute joints in mechanical systems is numerically efficient because the pressure distribution does not need to be evaluated. Furthermore, the methodology is easy and straightforward to implement in a computational code because resultant forces due to the fluid action are in explicit form. Some numerical difficulties can be observed if either the fluid viscosity is very low or the radial clearance of the journal-bearing is too large, which leads to large eccentricities and consequently the system becomes stiff. These difficulties are clearly associated with the limitations of the hydrodynamic lubrication theory.

\section{Acknowledgements}

The research work presented here was supported by Fundação para a Ciência e a Tecnologia and partially financed by Fundo Comunitário Europeu FEDER under project POCTI/EME/2001/38281, entitled 'Dynamic of Mechanical Systems with joint Clearances and Imperfections'.

\section{References}

[1] O.A. Bauchau, C. Ju, Modeling friction phenomena in flexible multibody dynamics. Computers Methods in Applied Mechanics and Engineering, 195 (2006) 6909-6924.

[2] C.A. Saracibar, M. Chimenti, On the Numerical modeling of frictional wear phenomena. Computers Methods in Applied Mechanics and Engineering, 177 (1999) 401-426. 
[3] P. Flores, J. Ambrósio, Revolute joints with clearance in multibody systems.

Computers and Structures, 82/17-19 (2004) 1359-1369.

[4] P.Flores, J. Ambrósio, J.C.P. Claro, H.M. Lankarani, Dynamics of Multibody Systems with Spherical Clearance Joints. Journal of Computational and Nonlinear Dynamics, 1(3) (2006) 240-247.

[5] O.A. Bauchau, J. Rodriguez, Modelling of Joints with Clearance in Flexible Multibody Systems. International Journal of Solids and Structures, 39 (2002) 41-63.

[6] P. Flores, J. Ambrósio, J.P. Claro, Dynamic Analysis for Planar Multibody Mechanical Systems with Lubricated Joints. Multibody System Dynamics, 12 (2004) $47-74$.

[7] J. Ambrósio, Efficient kinematic joint description for flexible multibody systems experiencing linear and nonlinear deformations. International Journal of Numerical Methods in Engineering, 56 (2003)1771-1793.

[8] S. Dubowsky, J.F. Deck, H. Costello, The Dynamic Modeling of Flexible Spatial Machine Systems with Clearance Connections. Journal of Mechanisms, Transmissions, and Automation in Design, 109 (1987) 87-94.

[9] T. Kakizaki, J.F. Deck, S. Dubowsky, Modeling the Spatial Dynamics of Robotic Manipulators with Flexible Links and Joint Clearances. Journal of Mechanical Design, 115 (1993) 839-847.

[10] P. Ravn, A Continuous Analysis Method for Planar Multibody Systems with Joint Clearance. Multibody System Dynamics, 2 (1998)1-24.

[11] R.S. Haines, A Theory of Contact Loss at Resolute Joints with Clearance. Proceedings of The Institution of Mechanical Engineers, Journal of Mechanical Engineering Science, 22(3) (1980) 129-136. 
[12] R.J. Roger, G.C. Andrews, Dynamic Simulation of Planar Mechanical Systems With Lubricated Bearing Clearances Using Vector-Network Methods. Journal of Engineering for Industry, 99(1) (1977) 131-137.

[13] T.S. Liu, Y.S. Lin, Dynamic Analysis of Flexible Linkages with Lubricated Joints. Journal of Sound and Vibration, 141(2) (1990) 193-205.

[14] A.L. Schwab, J.P. Meijaard, P. Meijers, A comparison of revolute joint clearance model in the dynamic analysis of rigid and elastic mechanical systems. Mechanism and Machine Theory, 37 (9) (2002) 895-913.

[15] H. Moes, E.G. Sikkes, R. Bosma, Mobility and Impedance Tensor Methods for Full and Partial-Arc Journal Bearings. Journal of Tribology, 108 (1986) 612-620.

[16] J.F. Booker, Dynamically Loaded Journal Bearings: Mobility Method of Solution. Journal of Basic Engineering, 4 (1965) 537-546.

[17] J.F. Booker, Dynamically Loaded Journal Bearings: Numerical Application of Mobility Method. Journal of Lubrication Technology, 1, (1971) 168-176.

[18] P.K. Goenka, Analytical Curve Fits for Solution Parameters of Dynamically Loaded Journal Bearings. Journal of Tribology, 106 (1984) 421-428.

[19] B.J. Hamrock, Fundamentals of Fluid Film Lubrication, McGraw Hill, New York, 1994.

[20] J. Frêne, D. Nicolas, B. Degneurce, D. Berthe, M Godet, Hydrodynamic Lubrication - Bearings and Thrust Bearings, Elsevier, Amsterdam, The Netherlands, 1997.

[21] O. Pinkus, S.A. Sternlicht, Theory of Hydrodynamic Lubrication, McGraw Hill, New York, 1961.

[22] G.B. Dubois, F.W. Ocvirk, Analytical Derivation and Experimental Evaluation of Short-Bearing Approximation for Full Journal Bearings, NACA, Report 1157, 1953. 
[23] A. Sommerfeld, Zur Hydrodynamischen Theorie der Schmiermittelreibung, Z. Angew. Math. Phys., 50 (1904) 97-155.

[24] P.E. Nikravesh, Computer Aided Analysis of Mechanical Systems, Prentice Hall, Englewood Cliffs, New Jersey, 1988.

[25] J. Baumgarte, Stabilization of Constraints and Integrals of Motion in Dynamical Systems. Computer Methods in Applied Mechanics and Engineering, 1 (1972) 1-16. [26] R.M. Phelan, Fundamentals of Mechanical Design, 3rd ed., McGraw-Hill, New York, 1970.

[27] ESDU 84031 Tribology Series, Calculation methods for steadily loaded axial groove hydrodynamic journal bearings. Engineering Sciences Data Unit, London, England, 1991.

[28] H. Rahnejat, Multi-body dynamics: historical evolution and application. Proceedings of the Institution of Mechanical Engineers, Journal of Mechanical Engineering Science, 214(C) (2000) 149-173.

[29] Flores, P., Dynamic Analysis of Mechanical Systems with Imperfect Kinematic Joints, Ph.D. Dissertation, University of Minho, Guimarães, Portugal, 2005. 